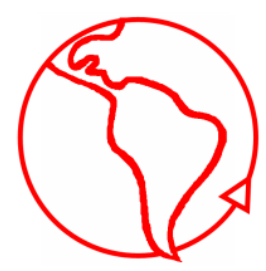

\title{
Lutero y el luteranismo: El buzón postal Martín Lutero
}

\author{
Luther and Lutheranism: The P.O. Box Martin Luther
}

Marco Ornelas

Departamento de Ciencias Sociales y Políticas, Universidad Iberoamericana, México

RESUMEN

Este artículo explora la idea de que la comunicación social opera de manera acoplada con las conciencias de los seres humanos -pero no es generada por ellas-, y utiliza como ejemplo al reformador alemán Martín Lutero. El traslape de operaciones de la conciencia y de operaciones comunicativas interpenetración- se hace posible por la distinción persona/actor, que se actualiza en la comunicación mediante esquemas (el esquema persona y el esquema actor), el llamado buzón postal Martín Lutero. La postura de Lutero ante las principales controversias en que participó -las rebeliones campesinas (anabaptismo radical), el libre albedrío y el debate sacramental-, marcó el futuro de su movimiento de Reforma y estuvo orientada decisivamente por el esquema persona. El esquema persona y el esquema actor también permite diferenciar entre Lutero/persona y el luteranismo/doctrina.

PALABRAS ClAVE: teoría de sistemas sociales; acoplamiento conciencia/comunicación; distinción persona/actor; esquemas; Martín Lutero

\section{ABSTRACT}

This article explores the idea that social communication performs coupled with the consciousness of human beings -but it is not generated by it-, and gives the example of the German reformer Martin Luther. An overlap in the operations of consciousness and of communication -interpenetration- is made possible with the person/actor distinction, which actualizes in communication through schemata (the person-scheme and the actor-scheme), the so called P. O. Box Martin Luther. Luther's positioning in relation to the polemical controversies in which he participated -the Peasants' War (radical Anabaptism), the free will, and the sacramental debate-, determined the future course of his Reform, and was decisively oriented by the person-scheme. The person/actor schemata also allow the distinction between Luther the person and Lutheranism the doctrine.

KEYWORDS: social systems theory; consciousness/communication coupling; person/actor distinction; schemata; Martin Luther

\section{INTRODUCCIÓN}

La teoría de sistemas sociales proporciona un enfoque novedoso para el estudio de las religiones al considerarlas estrictamente como comunicación re-producida en sociedad de manera acoplada con las conciencias de los seres humanos (Luhmann 1998, 2007a, 2007b). Para adentrarse en este entendimiento, se tomará el ejemplo del reformador alemán Martín Lutero. Siempre ha representado un reto explicar su

\section{REVISTA MAD - UNIVERSIDAD DE CHILE}

Revista del Magíster en Análisis Sistémico Aplicado a la Sociedad

Facultad de Ciencias Sociales, Departamento de Antropología.

Universidad de Chile

www.revistamad.uchile.cl 
distanciamiento de Erasmo de Rotterdam, a pesar del sustancial acuerdo en la significación de las comunicaciones que ambos actualizaron. También ha sido difícil comprender su renuencia a apoyar las sublevaciones campesinas (el anabaptismo radical de Carlstadt y Müntzer), no obstante haber sido él mismo quien inició la crítica frontal de la iglesia en un terreno institucional y teológico.

La teoría de sistemas sociales está provista del arsenal teórico y conceptual necesario para distinguir la comunicación doctrinal luterana de las acciones y determinaciones de Lutero (o viceversa), sin confundir los dos niveles -comunicaciones y conciencias- a los que considera intransparentes (clausurados en su operación); de otro modo, resultaría que las acciones de Lutero conllevaron "efectos no deseados", o de plano que, en el nivel de los motivos declarados, Lutero "fracasó" en su intento de reformar desde dentro y mantener unida a la cristiandad, en línea con las conclusiones a las que llega una de las biografías clásicas del reformador alemán (cf. Febvre 1988: 264 y ss.).

No es que se crea que los efectos no deseados o las motivaciones de la acción sean una mala explicación; sucede que ya hay una teoría que permite analizar y proporcionar explicaciones a fenómenos complejos como este. Ahora se puede decir: es la comunicación la que hace atribuciones internas (la acción individual con sus motivos) o externas (las vivencias no atribuibles a la acción intencionada), en tanto que las conciencias individuales solo participan de manera acoplada en el proceso al articular lingüísticamente sus pensamientos. ${ }^{1}$

\section{LA DISTINCIÓN CONCIENCIA/COMUNICACIÓN Y EL CONCEPTO DE INTERPENETRACIÓN}

Como bien se sabe, la teoría de sistemas sociales considera que los seres humanos son parte del entorno de los sistemas sociales, pero no forman parte de los mismos. Los sistemas sociales están formados exclusivamente por comunicaciones. Las comunicaciones, claro está, necesitan de seres humanos que las actualicen, de la misma manera que los pensamientos necesitan de una base neurofisiológica que les sirva de plataforma. Lo importante aquí es apuntar que la comunicación, las conciencias y

\footnotetext{
${ }^{1}$ Para esto véase Luhmann (1998: 140 y ss., 2007b: 258 y ss.). Ni siquiera el paso de una historia intelectual a una historia de las mentalidades, y luego a una historia de las ideas (Chartier 1992: 13 y ss.), como hace la Escuela de los Annales, logra desprenderse de la noción durkheimiana de que la sociedad está en las representaciones colectivas y no en la comunicación que se actualiza.
} 
los organismos son mutuamente intransparentes; todos ellos son considerados por la teoría como sistemas autopoiéticos clausurados en su operación. Y "el acoplamiento estructural ordinario entre sistemas de conciencia y sistemas de comunicación se hace posible por el lenguaje" (Luhmann 2007b: 79).

No hay una salida de continuidad entre las operaciones de los organismos (la reproducción de la vida), de las psiques (los pensamientos) y de los sistemas sociales (las comunicaciones). Una manera de explicar esto sería decir que la actividad consciente de los seres humanos se ha realizado por milenios sin necesidad del más mínimo entendimiento de las bases neurofisiológicas que la hacen posible. Trabajan en "paralelo" con el feliz desconocimiento de sus respectivas bases de funcionamiento:

El hombre no es ni siquiera capaz de observar por sí mismo lo que sucede dentro de él respecto de procesos físicos, químicos y vitales. Su sistema psíquico no tiene acceso a la vida, requiere de comezón, el dolor o cualquier otro medio para llamar la atención, con el propósito de provocar que opere otro nivel de conformación de sistemas, la conciencia del sistema psíquico. (Luhmann 1998: 61)

Esta misma intransparencia es supuesta en el funcionamiento de las conciencias y de las comunicaciones. El concepto de interpenetración denota esta intransparencia fundamental entre los sistemas sociales y los sistemas psíquicos, a pesar de que sus operaciones respectivas, las comunicaciones y los pensamientos, se desenvuelvan en el medio del sentido (Luhmann 1998: 77 y ss.). La idea de que el sentido es un medio compartido por las comunicaciones y las conciencias es lo que permite imaginar un traslape entre secuencias de pensamiento y secuencias comunicativas (acoplamiento que se hace posible por medio del lenguaje), precisamente lo que se quiere apuntar con el concepto de interpenetración.

La teoría prevé que los cuerpos o las conciencias puedan irritar o interferir la comunicación, pero nunca que la puedan determinar. Las conciencias no comunican (ni las comunicaciones piensan), solo las comunicaciones comunican (y solo las psiques piensan).

Si las comunicaciones se reproducen autopoiéticamente, mediante recursiones, forman una realidad emergente sui generis. No es el ser humano el que puede comunicar; solo la comunicación puede comunicar. Así como los sistemas de comunicación son sistemas operativamente clausurados que no pueden tener contacto con otros, también lo son los sistemas de conciencia -como por otro lado asimismo los cerebros, las células, etcétera. No existe comunicación -de conciencia a conciencia- que no sea socialmente mediada, así como no existe 
comunicación entre individuo y sociedad. Si quiere comprenderse de forma muy precisa la comunicación hay que excluir tales posibilidades y aun la otra posibilidad que consiste en concebir a la sociedad como espíritu colectivo. Solo la conciencia puede pensar, pero no puede pensar con pensamientos propios dentro de otra conciencia. Y solo la sociedad puede comunicar. En los dos casos se trata de operaciones propias de un sistema clausurado operativamente, determinado por la estructura (Luhmann 2007b: 76).

Así, la interpenetración es el concepto que denota que todo evento $\mathrm{t}_{1}$ en una línea del tiempo es resultado del acoplamiento lingüístico de comunicación y conciencias; la comunicación pone su complejidad a disposición de las conciencias y viceversa por intermedio del lenguaje. ${ }^{2}$ Este concepto se muestra gráficamente en la Figura 1 (la explicación de los esquemas contenidos en él sigue a continuación).

FIGURA 1. EL CONCEPTO DE INTERPENETRACIÓN CON ESQUEMAS

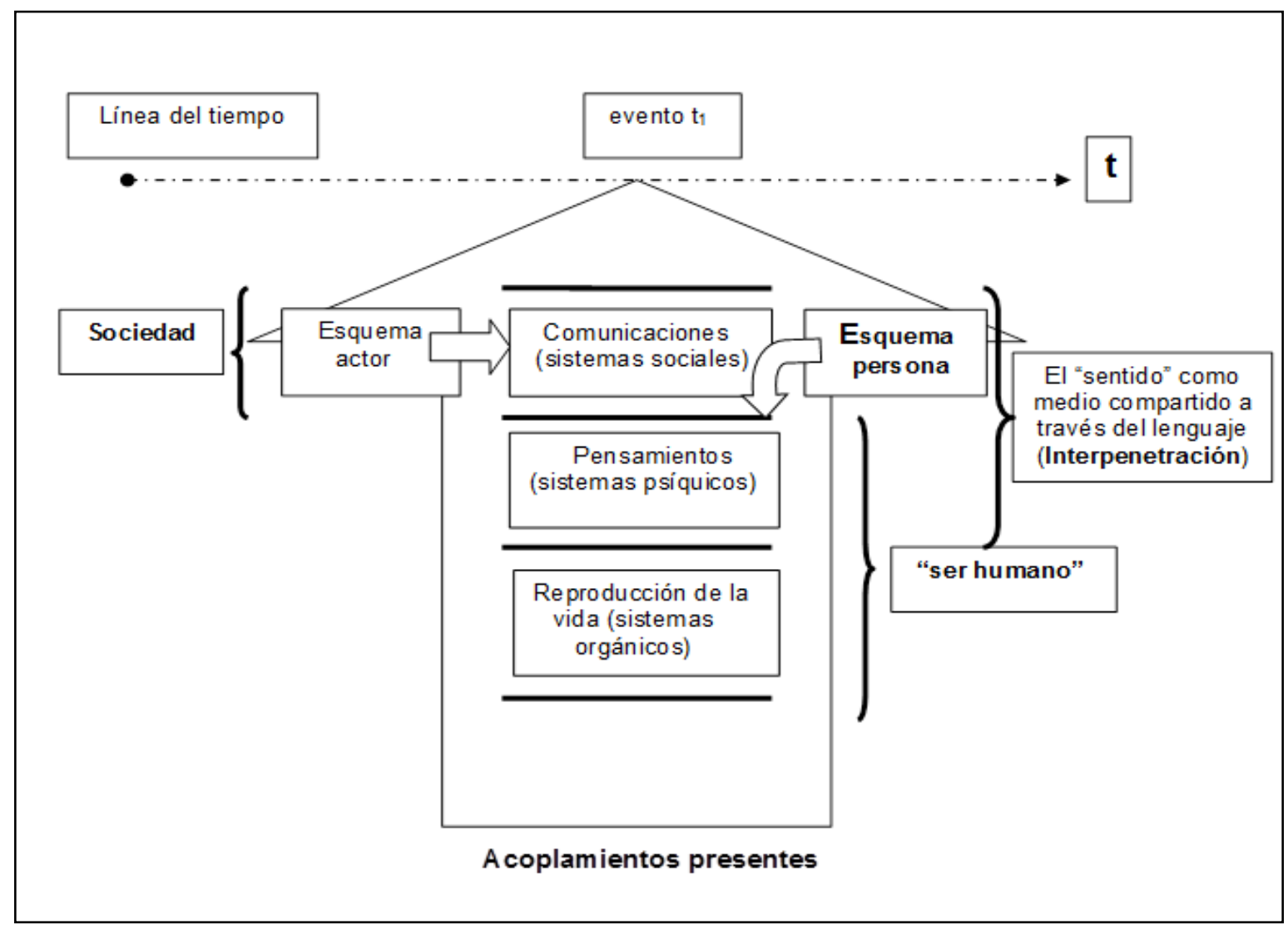

FUENTE: Elaboración propia

\footnotetext{
${ }^{2}$ En James (2002) puede encontrarse un largo y variado estudio de casos de interpenetración entre las conciencias y la comunicación religiosa que se tematiza como experiencia religiosa.
} 
2. LOS ESQUEMAS: EL BUZÓN POSTAL MARTÍN LUTERO.

A continuación se pretende proporcionar una explicación de la manera en que la comunicación se empalma con la facultad consciente de los seres humanos. Otra forma de plantear la situación sería preguntarse: ¿Cómo se habrá de entender la interpenetración entre la conciencia y la comunicación en el caso del iniciador de la reforma protestante? O en otros términos: ¿Cómo es imaginable este traslape entre secuencias de pensamiento y secuencias comunicativas en el caso de Martín Lutero? La respuesta que se propone en este artículo considera como fundamental la distinción persona/actor (Japp 2011).

En una formulación resumida, la distinción persona/actor se actualiza en la comunicación social mediante esquemas (esquema persona/esquema actor), que permiten a la comunicación una doble operación: mediante el esquema persona se "da oportunidad a la comunicación de reducirse a acciones y a la conciencia, de comprometerse con la persona" (Japp 2011: 176), mientras que el esquema actor permite que la comunicación se oriente plenamente hacia la sociedad y sus sistemas funcionales.

Los esquemas son reducciones de contextos complejos de acontecimientos. Un esquema descarga la comunicación (y la conciencia) de las exigencias de racionalización y extrae del no-saber modelos conocidos, generadores de significados. Especialmente las situaciones dotadas de una pluralidad de significados (interpretaciones incompatibles de un hecho) y de desconocimiento (no-saber) desatan la movilización de esquemas, los cuales se enlazan a algo ya conocido. (Japp 2011: 174-175) ${ }^{3}$

Esta propuesta teórica es contraintuitiva precisamente porque:

En el lenguaje ordinario se parte -como cosa sobreentendida- de los actores, en el sentido de individuos que actúan. Estos tienen motivos y actúan en consecuencia... Esto que para la praxis cotidiana es una unidad compacta de percepción (pensamiento, participación, entendimiento, acción e incluso individuo que actúa), en la perspectiva de la teoría sociológica de sistemas se disuelve precisamente en la diferencia entre conciencia y comunicación. (Japp 2011: 169)

\footnotetext{
${ }^{3}$ Los esquemas se apoyan a su vez en tipificaciones e idealizaciones para enfrentar las situaciones más diversas. Para esto revísese Schützeichel (2012: 75 y ss.), en el capítulo 6: Schütz, Luckmann y la objetivación del sentido.
} 
Sin embargo, no hay que olvidar que aunque se hable de actores, estos no dejan de representar atribuciones que hace la propia comunicación para autodirigirse:

Desde la perspectiva de la teoría sociológica de sistemas, el actor... es un domicilio social que la misma comunicación discurre con el fin de autodirigirse. La comunicación se resume en acciones sobre todo por la responsabilidad de sus consecuencias... Podría decirse también que la fluidez de la comunicación... adquiere la capacidad de enlace reduciéndose a la acción... Se percibe que los domicilios tienen nombres y que remiten a individuos: si comunicativamente se les toma en cuenta, se vuelven, de manera automática, individuos, se vuelven personas, aunque en la comunicación ellas no se toman como sujetos con total validez, sino como "expectativas de conducta atribuidas individualmente", esto es, como unidades de comunicación. (Japp 2011: 170171)

Es así que las personas ${ }^{4}$ con nombre y apellido (Martín Lutero) pueden ser consideradas como buzones postales en los que la comunicación deposita propósitos, motivaciones y logros (o fracasos); o tal vez valiera decir aquí que "las 'personas' están acopladas firmemente a la conciencia y los 'actores', débilmente. O, también, que los actores son domicilios generales estandarizados, mientras que las personas, domicilios individualizados que uno conoce" (Japp 2011: 171, n. 5). La distinción persona (con quien la conciencia debe tratar)/actor (enfilado hacia la comunicación) podría explicarse entonces de la siguiente manera:

Nadie designa a una persona que conoce más o menos bien como actor. Por el contrario, hablamos de actores cuando nos referimos a quienes desempeñan roles, cuando a pesar de que anteceda un escaso o nulo conocimiento personal se les atribuye capacidad de acción, porque en principio podemos obtener este conocimiento de la persona. Lo normal es que se use un concepto de actor dirigido a eliminar la indeterminación de la comunicación... Los actores aseguran a la comunicación la capacidad de enlace, simplificando una determinada atribución de la acción, la cual, a su vez, puede comunicarse. Los actores llevan, por una parte, una vida entre individuos de alguna manera señalados y, por otra, una comunicación general con capacidad de acción o incluso de domicilio. (Japp 2011: 171)

\footnotetext{
${ }^{4}$ No debe confundirse a los seres humanos de carne y hueso con las personas. Las personas son un constructo más de la comunicación: "Las personas surgen, entonces, mediante la participación de los seres humanos en la comunicación. Satisfacen las necesidades del observar, en cuanto se les atribuye consistencia de opiniones y actitudes, perseverancia de comportamiento, intereses propios calculados en perspectiva, etc. Ellas no viven, no piensan; son construcciones de la comunicación para fines comunicativos" (Luhmann 2010: 117-118).
} 
Es esta oscilación entre la persona y el actor la que permite plantear como esencial para el acoplamiento entre conciencia y comunicación al esquema persona: "Puede verse -a partir de que el individuo permanece conocido como persona en lo que es- que el esquema persona (a diferencia del de actor) posibilita el acoplamiento estructural entre individuo y sociedad" (Japp 2011: 174). La importancia del esquema persona estriba precisamente en que "comprende una pequeña parte de las operaciones de la conciencia y una pequeña parte de las operaciones comunicativas incluyendo aquella delimitada por las expectativas personales-. Si esto no fuera así, se daría al traste con la autonomía de los sistemas involucrados (se llegaría a personas sobresocializadas o a una comunicación sobrepersonalizada)" (Japp 2011: 179).

3. LAS REBELIONES CAMPESINAS (ANABAPTISMO RADICAL), EL LIBRE ALBEDRÍO Y EL DEBATE SACRAMENTAL.

¿Pudieran encontrarse ejemplos de la manera en que el esquema persona operó en Martín Lutero? Se pueden señalar al menos tres, todos cruciales para el devenir del movimiento de Reforma: su deslinde de las rebeliones campesinas de 1524-25; en relación con estas, su desmarcaje del anabaptismo radical de Carlstadt y Müntzer ${ }^{5}$, finalmente, su relación polémica y final ruptura con Erasmo de Rotterdam primero, y con Zwinglio y Ecolampadio después.

En relación con las rebeliones campesinas de 1524-1525, se coincide en aceptar que estas señalaron el punto de quiebre en la obra de Lutero entre el reformador polemista, agitador, y uno más enfocado a organizar la naciente comunicación no conformista (Egido 2001: 15 y ss.; Edwards 2003: 192). ¿Cómo pudo aquí la biografía traslapar a la comunicación social (y viceversa)? Hacia 1510, de vuelta de Wittenberg a la universidad de Erfurt, su Alma Mater, Lutero enfrentó una revuelta campesina que ejecutó al presidente del concejo de la ciudad y quemó el vestíbulo de la universidad con su biblioteca. Lutero nunca perdonó esta violencia intolerante que permite explicar por qué años después, al momento de la guerra del campesinado, tomó una postura cautelosa primero y decisivamente opuesta después (Atkinson 1980: 66 y ss.). Datos biográficos variados permitirían este juego entre el esquema persona y el esquema actor, de tal

\footnotetext{
${ }^{5}$ Sobre la controvertida historiografía de Thomas Müntzer, véase Friesen (1974).
} 
manera que la conciencia y la comunicación quedan intercaladas (no transparentadas). ${ }^{6}$

De alguna manera, la opción finalmente tomada por Lutero respecto de las sublevaciones campesinas, terminó por apartarlo de Carlstadt, su colega en la Universidad de Wittenberg. Hay quienes reducen el radicalismo de Carlstadt a su capacidad para sacar las consecuencias prácticas de los planteamientos teológicos de Lutero. En esa misma medida, Carlstadt vendría siendo un importante promotor de los planteamientos reformistas radicales, en especial de los anabaptistas:

Fue Carlstadt quien tradujo las afirmaciones de Lutero en un marco teológico coherente, quien proporcionó la enseñanza teológica y quien sacó las consecuencias de los pronunciamientos de Lutero. Es así que uno pudiera entender a Carlstadt como precursor de Melanchton, quien realizó funciones similares en años posteriores guiado por una muy diferente propensión teológica... Si el lugar eminente ocupado por Carlstadt en los primeros años de la Reforma debe atribuirse a su habilidad para discernir algunas consecuencias o ramificaciones del pensamiento de Lutero, entonces esta misma afirmación caracteriza su involucramiento con el apogeo de la "reforma radical". (Hillerbrand 1966: 395-396) ${ }^{7}$

Por último, está claro que el Renacimiento y la Reforma tuvieron muchos puntos de convergencia. Ambos movimientos estuvieron interesados ante todo en restaurar el cristianismo mediante una reforma de la iglesia: "Tanto Erasmo como Lutero se preocuparon por restaurar el cristianismo y por reformar a la iglesia; difirieron en sus interpretaciones sobre la esencia del cristianismo y en las formas de llevar a cabo la Reforma" ${ }^{8}$ (Kleinhans 1970: 460). O articulado con otras palabras:

Había muchos puntos de contacto entre ambas tendencias: anhelo de reforma, preocupación por las bellas letras, oposición a formas romanas, enemistad cordial con la escolástica, atención primordial a la fuente de la Escritura,

\footnotetext{
${ }^{6}$ Entre ellos, haber quedado sobrecogido al ser sorprendido por una tormenta eléctrica a campo abierto, y que lo llevaría a prometer dedicar su vida a Dios; o también, para explicar su aversión por todo lo romano, incluido el papa, el viaje realizado durante un mes a Roma en 1510. Lo relevante del mismo no es tanto lo que vio ahí, sino que lo realizaba en calidad de emisario de las casas agustinas de Erfurt y Nüremberg: intentaba negociar con Roma que la disciplina de las casas no fuera tocada, pues ya habían sido reformadas. Aunque en realidad "no era la reforma lo que interesaba al pontificado, sino la política y la guerra, la música y la caza, las comedias y el carnaval" (Atkinson 1980: 68). De su viaje a Roma y otros muchos detalles biográficos véase también a Febvre (1988).

${ }^{7}$ Las traducciones españolas de artículos en inglés son del autor.

${ }^{8}$ El punto de doctrina que terminó por separarlos fue el entendimiento en torno al libre albedrío defendido por Erasmo e inaceptable para la teología de la cruz de Lutero-.
} 
maneras críticas, espiritualidad más directa y personal, etc. Si Lutero no se hubiera lanzado por la vía revolucionaria, o si no le hubieran lanzado los hombres y las circunstancias, la conexión humanismo-Reforma hubiera encauzado las cosas por otros derroteros. Sin embargo, en profundidad, corrían torrentes irreductibles y que se distanciaron cuando Lutero expresó con claridad su concepción del hombre, de la libertad, cuando evidenció su dogmatismo. Humanistas agudos -y Erasmo lo era- se percataron de que se trataba de sustituir una autoridad por otra y -más decisivo- de que en el sistema luterano no cabía el hombre como protagonista. (Egido 2001: 28-29)

Llama la atención la forma en que se concluye de este distanciamiento $y$, sobre todo, las etiquetas sobrepuestas a cada uno de los dos bandos: "La inteligencia era imposible entre dos personas que parten de presupuestos básicamente divergentes, como era el moderado pelagianismo 9 de Erasmo y el agudo maniqueísmo agustiniano ${ }^{10}$ de Lutero" (Egido 2001: 29). Aquí más bien se postula que la comunicación dogmática per se, sin estar del todo ausente, no es el factor primordial en la explicación. ${ }^{11}$ Lo que los separa son más bien rasgos biográficos, el esquema persona, la conciencia que se compromete con la persona, trátese del espíritu libertario humanista del uno o de la tozudez teológica del clérigo agustino. $\mathrm{Y}$ algo muy parecido podría sostenerse para el diferendo Lutero/Zwinglio. ${ }^{12}$

El alejamiento de Lutero y Erasmo fue el preludio de la disputa en torno a la cena del Señor con Zwinglio. Aquí vuelve a llamar la atención que para explicar las distintas concepciones de la presencia de Cristo en la cena, se vuelva a echar mano de la comunicación no conformista de largo

\footnotetext{
${ }^{9}$ Doctrina que niega el pecado original e inclusive la necesidad del bautismo y de la gracia. Los principios morales y la disciplina monástica inclinan al hombre a la virtud y le permiten acceder a la perfección que le libera del pecado (Mitre 2000: 41).

${ }^{10}$ El maniqueísmo es una religión que opone dos principios, uno de luz y bondad (Dios) a otro de maldad y oscuridad (materia). Tiene como antecedente las doctrinas del gnosticismo (Mitre 2000: 12; Markschies 2002: 37-38). Aquí, Egido se refiere a la doctrina agustiniana que contrapone los dos reinos (el reino terrenal al reino de los cielos) expuesta por san Agustín en La ciudad de Dios. Debe recordarse que antes de militar en el cristianismo, Agustín de Hipona fue maniqueo.

${ }_{11}$ Por si cupiera duda de la importancia de Erasmo para la Reforma: "Erasmo hizo más que ninguno de sus contemporáneos por promover la causa de la Reforma, por una parte con su trabajo positivo de revivir los estudios clásicos, bíblicos y patrísticos, y por otro, con su obra negativa de denunciar los abusos eclesiásticos, la ignorancia y mojigatería de los frailes y el oscurantismo intelectual de los profesores... Fue uno de los hombres de transición, como Reuchlin o Staupitz, solo que mayor y más importante. Fue el Juan Bautista del nuevo movimiento" (Atkinson 1980: 261).

12 Por ejemplo, si bien Lutero aceptaba la presencia real así como que se creyese que el pan y el vino eran solo eso: pan y vino (Egido 2001: 95), se opuso a que Zwinglio dijera esto mismo (que el pan y el vino eran solo eso: pan y vino) y lo llamara presencia simbólica. Véase infra.
} 
aliento. ${ }^{13}$ Mientras Lutero era eutiquiano (monofisita), ${ }^{14}$ Zwinglio mostraba tendencias nestorianas: ${ }^{15}$

La teología de Lutero es casi aquí eutiquiana en su cristología: con lenguaje poco técnico, casi admitía que lo divino y lo humano se interpenetraban; pensaba en Cristo como presente en todas partes en el sacramento con su cuerpo humano transfigurado. En cierto sentido, la cristología de Zwinglio es "nestoriana" en esta cuestión; sin usar términos técnicos, diremos que se resistía a adoptar la misma idea de la unión de dos naturalezas en los elementos consagrados... Parecía que Lutero quería intensificar la realidad de la naturaleza divina en Jesús, el hombre, que se había rebajado a asumir nuestra humanidad... Zwinglio, coincidiendo en esto con Erasmo, recalcaba la humanidad de Jesús (aunque no ponía en duda su divinidad). (Atkinson 1980: $307 \mathrm{ss})^{16}$

Sería bastante aventurado tratar de delinear aquí fronteras precisas entre la persona y el actor del buzón postal Martín Lutero; la intención última es sugerir y hacer evidentes algunos contornos... con ayuda de la teoría. A decir de los especialistas, Lutero tuvo fobias que rayaban en la patología; los objetos de sus fobias: los judíos, los turcos, el papa y el demonio (Egido 2001: 53 y ss.). Aquí sirve de mucho una teoría social como la expuesta en los apartados anteriores: lo que se considera "rasgos" de personalidad, ${ }_{1}^{17}$ pudieran pensarse también como el rejuego del esquema persona (el buzón postal Martín Lutero) que no deja de orientarse por las características de las comunicaciones sociales del siglo xvi. Aparte de la acentuada proclividad a la superstición común entre personajes de la época, pudiera decirse que se trata de fobias características de aquellos tiempos (los judíos, por tradición; los turcos, por contestar y poner en duda la supremacía de la cristiandad; el papado, por estar desacreditado de por sí

\footnotetext{
${ }^{13}$ Sobre la comunicación no conformista y su importancia para la conformación de la dogmática cristiana, véase Ornelas (2011).

${ }^{14}$ El monofisismo es la doctrina que, si bien no niega la doble naturaleza del Hijo -humana y divina-, afirma que la naturaleza humana en Cristo habría sido absorbida por la divina (Mitre 2000: 21).

${ }^{15}$ El nestorianismo es la doctrina que considera a María como engendradora de Cristo, mas no así de Dios mismo (Mitre 2000: 23).

${ }^{16}$ En 1529, en Marburgo, Zwinglio y Lutero, con la mediación de Felipe de Hessen, asistieron a un coloquio con la intención de zanjar la disputa, sin éxito.

${ }^{17}$ El "carácter" atribuido a la persona y los motivos quedan conectados en una constelación que comunica de manera creíble: “Diremos que las personas (¡no los seres humanos!) tienen un carácter, a través del cual es posible regular qué motivos pueden ser adscritos, de manera más o menos plausible. La máquina histórica del sistema social puede cambiar los motivos que se adjudican a las personas. La simbología persona/motivo dota al sistema social de una combinación entre continuidad y discontinuidad, esto es, de la posibilidad de transformaciones limitadas y controlables. Y, todo esto, en un ambiente psíquico-orgánico que permanece inaccesible" (Luhmann 2010: 123).
} 
y cuestionado por el humanismo renacentista; el demonio, por razones obvias). Todas ellas, con excepción de la fobia al papado, seguramente fueron compartidas por los papas mismos... En cambio, donde se acierta es al dar testimonio de su humor: "Su carácter sajón se abre en la embestida, en el chiste, en la broma constante... Su teología se basa en reírse del hombre, reducido a una piltrafa, y en el gozo del encuentro con y de Dios en Cristo, causa de alegría, en contraste con la angustia medieval de que partió" (Egido 2001: 59).

La ira/enojo incontrolable de Lutero, parecieron acompañarlo durante toda su vida, en particular hacia el final:

Una lectura de los textos polémicos de Lutero deja la impresión nítida, sin embargo, de que en los últimos años su enojo se tornó más agudo y menos movido por la compasión, el humor o la reflexión teológica. Más todavía, su perenne lenguaje mordaz se hizo más ordinario y escatológico. Los objetos de su ira se volvieron bajo su pluma en los hipócritas más viles, totalmente torcidos y falsos, comparsas serviciales del demonio que merecen la suerte más espantosa. (Edwards 2003: 204)

Estos señalamientos (Edwards 2003: 204) no solo se refieren a la salud psicológica de Lutero, sino también a su salud físico-orgánica, al grado de aventurar un síndrome de Ménière acompañado con senilidad. Se está de nuevo frente al concepto de interpenetración y a los acoplamientos presentes en todo evento comunicativo: orgánico/psíquico/social.

\section{CONCLUSIONES}

La teoría de sistemas sociales ha permitido abordar la diferencia entre Lutero/persona y el luteranismo/doctrina con base en el juego oscilante entre el esquema persona y el esquema actor: el llamado buzón postal Martín Lutero. De esta manera permite proporcionar explicaciones posibilitadas por el traslape entre las operaciones de la conciencia y las operaciones comunicativas.

La postura de Lutero hacia las rebeliones campesinas, ante los anabaptistas e "iluminados" (señaladamente Carlstadt, aunque paradójicamente Lutero mismo pueda considerarse un "iluminado" más), y frente a Erasmo y Zwinglio estuvieron orientadas decisivamente por el esquema persona. Es así que las argumentaciones, motivos y decisiones de Lutero en estas materias (el rigorismo sin concesiones de anabaptistas, la polémica en torno al libre albedrío y el debate sacramental) estuvieron fuerte- 
mente determinados por la necesidad de compatibilizar la comunicación de su entorno social inmediato -la situación de Sajonia en la primera mitad del xvi y la circunstancia de ser un monje agustino de la universidad de Erfurt recién cobijado en Wittenberg- con expectativas altamente personalizadas, por decirlo así: biográficas.

El esquema actor, en cambio, determinaría los parámetros de la comunicación social cuyo principal referente es la tradición milenaria de la comunicación no conformista cristiana, decisivamente antijerárquica y antisacramental (piénsese en la tradición rigorista cristiana encarnada en el donatismo, los valdenses, el catarismo y los husitas checos). Es esta importante tradición la que alimentaría algunos puntos centrales de la doctrina luterana: el rechazo a la autoridad religiosa y la correspondiente máxima autoridad puesta en las Escrituras, el sacerdocio universal, la crítica a las indulgencias, la desconfianza extrema en los sacramentos, señales exteriores de la gracia.

Del luteranismo, pues, será posible diferenciar entre el escalamiento de la tradición herética cristiana, y la aportación de la persona a esta tradición (la justificación por la sola fe, punto central de la teología de la cruz de Lutero). Si este punto de teoría -el acoplamiento individuo/sociedades interpretado en esta forma, podrá verse qué tan lejos está la teoría de considerar la facultad consciente de los seres humanos como prescindible para la comunicación, aunque al mismo tiempo se niegue terminantemente a dar por supuesta la transparencia/homogeneidad entre el individuo y la sociedad, entre los sistemas psíquicos y los sistemas sociales, puesto que de otra manera quedaría cuestionada la autopoiesis de los sistemas involucrados. RM

\section{BIBLIOGRAFÍA}

Atkinson, J. (1980). Lutero y el nacimiento del protestantismo. Madrid: Alianza Editorial.

Chartier, R. (1992). El mundo como representación. Estudios sobre historia cultural. Barcelona: Gedisa.

Edwards, M. (2003). Luther's Polemical Controversies. En D. McKim (Ed.), The Cambridge Companion to Martin Luther (pp. 192-205). Cambridge: Cambridge University Press.

Egido, T. (2001). Lutero Obras. Edición preparada por Teófanes Egido. Salamanca: Sígueme.

Febvre, L. (1988). Martín Lutero: Un destino. México D.F.: Fondo de Cultura Económica. 
Friesen, A. (1974). Philipp Melanchthon (1497-1560), Wilhelm Zimmermann (18071878) and the Dilemma of Muntzer Historiography. Church History, 43(2), 164182.

Hillerbrand, H. (1966). Andreas Bodenstein of Carlstadt, Prodigal Reformer. Church History, 35(4), 379-398.

James, W. (2002). Las variedades de la experiencia religiosa: Estudio de la naturaleza humana. Barcelona: Península.

Japp, K. P. (2011). Actores políticos. En J. Torres-Nafarrate \& D. Rodríguez (Eds.), La sociedad como pasión. Aportes a la teoría de la sociedad de Niklas Luhmann (pp. 169-200). México D.F.: Universidad Iberoamericana.

Kleinhans, R. (1970). Luther and Erasmus, Another Perspective. Church History, 39(4), 459-469.

Luhmann, N. (1998). Sistemas sociales. Lineamientos para una teoría general. México D.F.: Anthropos/Universidad Iberoamericana/CEJA.

Luhmann, N. (2007a). La religión de la sociedad. Madrid: Trotta.

Luhmann, N. (2007b). La sociedad de la sociedad. México D.F.: Herder/ Universidad Iberoamericana.

Luhmann, N. (2010). Organización y decisión. México D.F.: Herder/ Universidad Iberoamericana.

Markschies, Ch. (2002). La gnosis. Barcelona: Herder.

Mitre, E. (2000). Las herejias medievales de Oriente y Occidente. Madrid: Arco Libros.

Ornelas, M. (2011). La importancia de las herejías para la conformación de una dogmática cristiana. En I. Farías \& J. Ossandón (Eds.), Comunicaciones, semánticas y redes. Usos y desviaciones de la sociología de Niklas Luhmann (pp. 85-104). México D.F.: Universidad Iberoamericana/ITESO.

Schützeichel, R. (2012). Teorías sociológicas de la comunicación. México D.F.: Manuscrito. [Traducción española de Javier Torres Nafarrate].

\section{SOBRE EL AUTOR}

Marco Ornelas es Becario Posdoctoral adscrito al Departamento de Ciencias Sociales y Políticas de la Universidad Iberoamericana, México. Doctor en Ciencias Sociales por El Colegio de Sonora, México. Entre sus líneas de trabajo se cuentan: sociología de la religión, teorías sociológicas de la comunicación, historia del cristianismo. Entre sus últimas publicaciones se destacan: La importancia de las herejías para la conformación de una dogmática cristiana [En I. Farías \& J. Ossandón (eds.), Comunicaciones, semánticas y redes. Usos y desviaciones de la sociología de Niklas Luhmann, México D.F., 2011], La sociología de la religión de Niklas Luhmann [En Niklas Luhmann: Sociología de la religión, México D.F., 2009. Autor del prefacio y compilador del libro], The Catholic Mass in a Secular World [Journal of Dharma, 2007].

\section{CONTACTO}

Universidad Iberoamericana, Ciudad de México,

Departamento de Ciencias Sociales y Políticas.

Prol. Paseo de la Reforma 880. Lomas de Santa Fe. 
C. P. 01219

México, D. F.

México

marcornelas@hotmail.com

Recibido: Mayo 2014

Aceptado: Agosto 2014 